\title{
Homer's alcohol connection
}

DOI:

10.1038/nrn2161
Many TV-watching readers will be familiar with a certain Homer and his relationship with alcohol. Now, Urizar et al. show that expression of a gene with the same name in a subset of neurons in the Drosophila ellipsoid body plays a part in the flies' sensitivity to and rapid tolerance for ethanol.

Flies grow tolerant to the effects of ethanol vapour after just one exposure, and this tolerance becomes more permanent after multiple exposures. In order to elucidate the molecular mechanisms underlying these adaptations, the authors used microarrays to identify Drosophila genes that show altered expression after one or multiple exposures to ethanol vapour. They found that ethanol changed the transcription of many genes, but the investigators focused on the homer gene. Homer is thought to be involved in the regulation of synaptic plasticity, and its expression decreased by $17 \%$ and $24 \%$ after single and repeated exposures, respectively, to ethanol.

To test whether this gene has a direct and functional role in ethanol-induced behaviours, the authors studied homer null-mutant flies. Exposure to ethanol sedated homer-null mutants 5 minutes earlier on average than wild-type flies. Moreover, a larger percentage of mutants were sedated by the alcohol fumes, indicating that homer mutants displayed increased sensitivity to ethanol. In addition, fewer mutant flies developed rapid tolerance on a second exposure to ethanol than wild-type flies. Homer mutants did not have altered ethanol absorption or metabolism, indicating that the role of homer in regulating sensitivity and rapid tolerance to ethanol is not related to alterations in ethanol levels.

The authors used the UAS/GAL4 system to re-introduce the wild-type homer gene into the CNS and PNS of the mutant flies. The resulting pan-neuronal expression of homer restored both ethanol sensitivity and rapid tolerance. But in which parts of the Drosophila brain is homer crucial for this rescue?

Using immunohistochemistry, Urizar et al. observed staining for the homer protein in most neuropil areas in the wild-type Drosophila brain, including the antennal lobes and the ellipsoid and fan-shaped bodies of the central complex. Next, the authors used different GAL4 lines to drive UAS-homer expression in the null flies in particular regions of the nervous system. Homer expression in most brain areas did not alter the flies' ethanol sensitivity and tolerance. However, gene expression in the R2 and R4 ring neurons - but not in other neurons - of the ellipsoid body resulted in significant rescue of ethanol sensitivity and of rapid tolerance.

This study provides evidence that regulation of sensitivity to and tolerance for ethanol in
Drosophila involves a small group of homer-expressing neurons in the ellipsoid body of the central complex, although the precise role of homer is still unclear. Homer is highly homologous to mammalian HOMER1; it remains to be seen whether this protein has a role in adaptation to alcohol in humans.

Leonie Welberg

ORIGINAL RESEARCH PAPER Urizar, N. L. et al Drosophila homer is required in a small set of neurons including the ellipsoid body for normal ethanol sensitivity and tolerance. J. Neurosci. 27, 4541-4551 (2007)

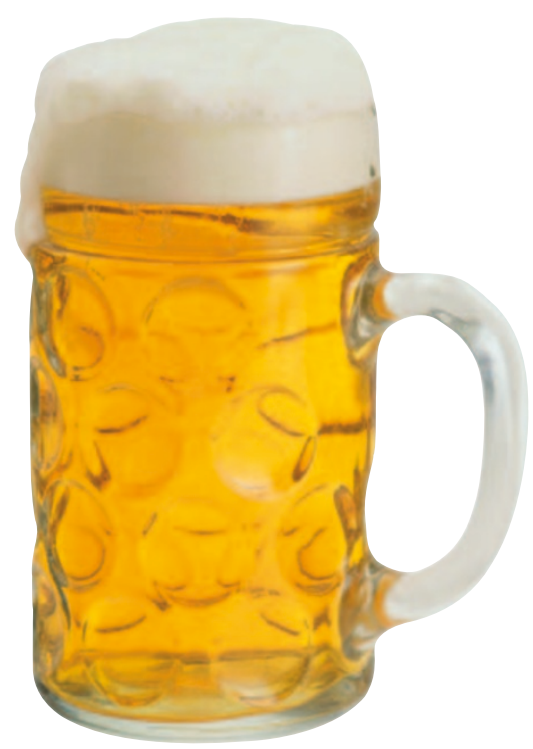

